\title{
Experimental research on tungsten alloy spherical fragments penetrating into carbon fiber target plate
}

\author{
Dou Hong a (D), Wen-bin $\mathrm{Li}^{\text {a }}$ (D), Yu Zheng ${ }^{\text {a* }}$ (D), Ning Jiang ${ }^{\text {a }}$ \\ ${ }^{a}$ Ministerial Key Laboratory of ZNDY, Nanjing University of Science and Technology, Nanjing, China. Email: 875854607@qq.com, \\ Iwb2000cn@njust.edu.cn, zhengyu@njust.edu.cn, njustjn@163.com
}

* Corresponding author

https://doi.org/10.1590/1679-78256510

\begin{abstract}
A ballistic projectile launching device was used to study the penetration behaviors of tungsten alloy spherical fragments of various diameters into carbon fiber composite target plates of different thicknesses. Based on the ballistic test results, we obtained the relationship between ultimate penetration velocity, target plate thickness, and fragment diameter as well as the relationship between the fragment penetration energy and fragment incident velocity. Using dimensional analysis, we obtained a formula relating the incident fragment velocity and the fragment penetration energy, which showed good agreement with the experimental values. We also analyzed the main fracture mode and the energy absorption mechanism of the carbon composite target plate under high-velocity impacts of tungsten alloy spheres and investigated the experimental damage modes of the target plate at different fragment velocities during the ballistic impact.
\end{abstract}

\section{Keywords}

tungsten alloy spherical fragments, carbon fiber composite target plates, penetration energy, energy absorption mechanism

\section{Graphical Abstract}

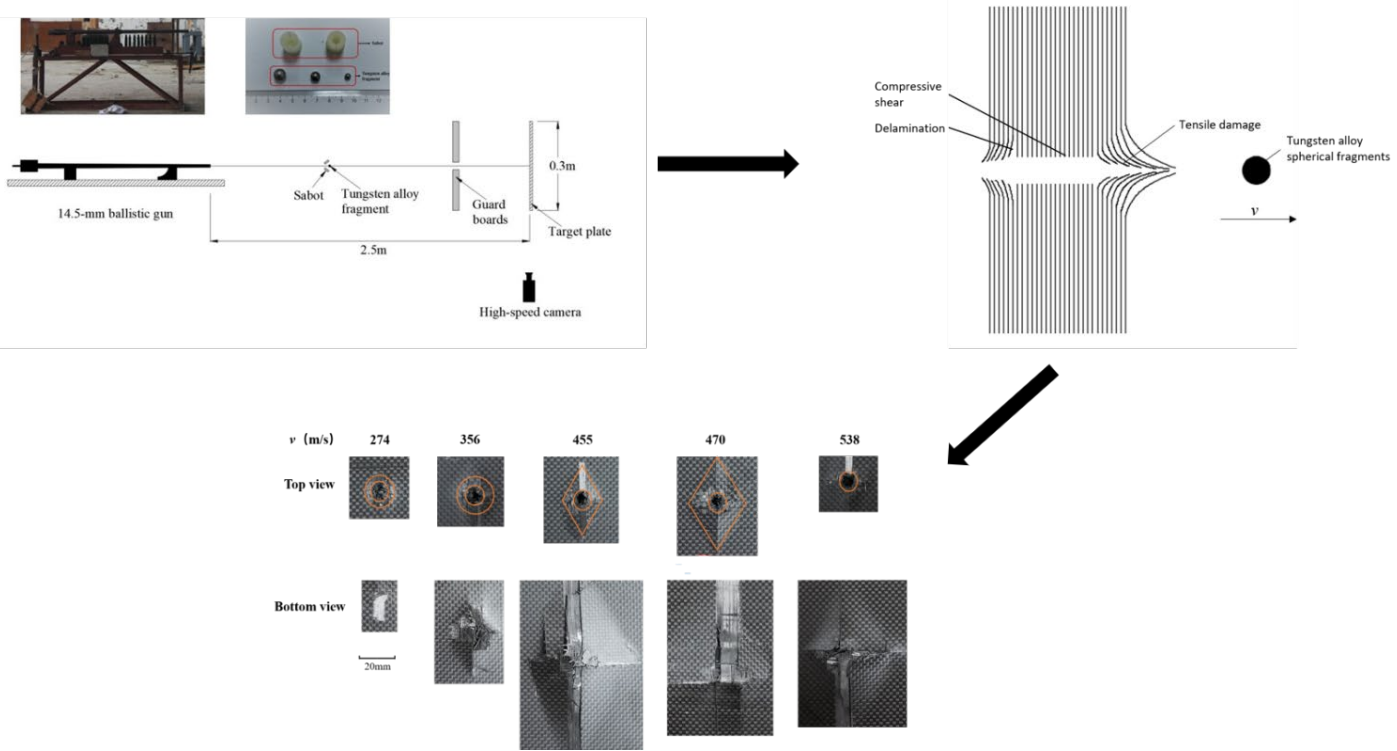




\section{INTRODUCTION}

In recent years, increasingly complex battlefield environments have created higher demands for the maneuverability of tanks and heavy armor, making lightweight and high-strength composite armor a research focus in the field of armored passive defense. Non-metallic composite materials have low densities, ultra-high strengths, and low costs, and they possess great application potential and prospects in the field of composite armor (Zhang F et al.,2015; Zhang and Dai, 2000) . At present, non-metallic composite materials have been widely used in the field of armor. Most of them are sandwiched between two layers of metal materials, or sandwiched between two layers of metal materials, which can improve the mobility of armored vehicles without reducing the defense capability of armor.Typical nonmetallic composite materials include rubber, ceramics, aramid fiber, and carbon fiber composite materials (Withers $J$ C et al.,2011; Meffert and Milewski, 2002) Among these, carbon fiber composite materials are highly favored in the field of composite armor due to their excellent tensile properties.

The study of the penetration resistance performances of carbon fiber composite materials under high-velocity impacts is highly important, since these materials are widely used as structural materials in the field of armor protection. Zhu et al. (1992) and Morye et al. (2000) studied the penetration process and perforation mechanism of fragments with different shapes impacting composite target plates. Wen (2000) established an analytical model to predict the lateral impact of rigid projectiles with different head shapes as well as the penetration and perforation of composite solid laminates. Pandya et al. (2013) conducted ballistic impact tests on different hybrid composite materials to study the damage modes of the target plates. In the study of low-velocity impact damage of targets, the size of the target will have a significant effect, but in high-velocity impacts, it is the local response of the target that matters, and the effect of the target size on the impact damage will be diminished (Cantwell and Morton, 1989; Chai and Zhu, 2011). Koubaa et al. (2017) studied the damage characteristics of aluminum target under low and medium velocity impact by numerical simulation, and qualitatively analyzed the influence of fragment head shape on the limit ballistic velocity. Mars et al. (2018a; 2018b) studied the low velocity impact of FGM and PA plates, and introduced the Mori-Tanaka model for the first time to analyze the micromechanics in the impact process.

In the study of the anti-penetration performances of carbon fiber target plates, the penetration process has been found to be affected by the masses, shapes, sizes, and velocities of the fragments, and the ballistic impact performances of the carbon fiber target plates are also affected by the geometries and mechanical properties of the plates. Researchers have also conducted extensive work on the damage characteristics of carbon fiber target plates under impacts. Wambua et al. (2007) gave a description of the failure mode of composite materials: after the initial damage caused by the shear force, the tensile load damages the material, causing delamination around the area under a high-velocity impact and apparent local bulging. Zukas (1990) proposed that the failure mode of the target be classified as brittle fracture, ductile hole growth, fragmentation, radial fracture, blockage, and petal-shaped damage. The visual characteristics of the hole were described through a series of images based on the failure mode. The study of Shaktivesh et al. (2013) showed that after fragments came in contact with a target plate, the contact force between the fragment and the target plate immediately generated a through-thickness shear stress in the target around the fragment. If the shear stress exceeded the shear strength of the target plate, the ply failed during shear, and those that did not fail experienced a tensile strain. On this basis, Razali et al. (2014) studied the relationship between the failure mode of the target plate and the energy absorption characteristics of the target plate. Kinloch et al. (1985) and Kinloch and Taylor $(2003 ; 2006)$ studied the fracture behavior of hybrid particle composites, improved the preparation method of epoxy micro nano composites, improved the modulus and fracture properties of materials, and studied the changes of properties of epoxy micro nano composites prepared under different silicate concentrations. Miglani et al. (2018) and Devarajan et al. (2020) studied the mechanical properties of Orthotropic Composite Laminates Based on the first-order shear deformation theory, and verified the results by numerical simulation. Along with the study of composite target plates, Hazell et al. (2008) found in experiments that the energy transferred from fragments to the target plate increased with increasing impact energy, which is consistent with the phenomenon studied by Wang et al. (2017) and S Ping et al. (2019)

With the exploration and study of the effect of fragments during the penetration of a target, the formula for the ultimate penetration velocity of the fragments has been gradually improved. Its range of application has been widened and its calculation accuracy has been improved. However, the greater the fragment energy is, the greater the energy required to penetrate the target plate becomes. Therefore, to characterize the penetration characteristics of fragments using the ultimate penetration velocity alone is still inadequate. All the above literatures about ballistic impact only qualitatively analyze the relationship between the energy required by the target plate and the velocity of fragment entering the target, and do not describe the relationship quantitatively. However, there is no description of the relationship between damage characteristics and impact velocity.

In this work, through the study of the penetration effect of carbon fiber target, the damage characteristics of carbon fiber target are analyzed, and the change trend of damage effect with the impact velocity of fragments is studied. By studying the effect of carbon fiber target plate penetration and using a dimensional analysis method, we determined the relationship between the fragment penetration energy of carbon fiber target plates and the fragment velocity. In 
addition, we conducted armor piercing tests using tungsten spheres of different diameters launched from a 14.5-mm ballistic gun and obtained good agreement between the test results and the calculated values.

\section{FIGURES}

\subsection{Test facility}

The experimental study was performed using a 14.5-mm ballistic gun impact test device. Figure 1 shows the layout of the ballistic impact test apparatus. The devices used in the test included a $14.5-\mathrm{mm}$ ballistic gun, a gun bolt, guard boards, a target plate, a holder frame for the target, and a high-speed camera.
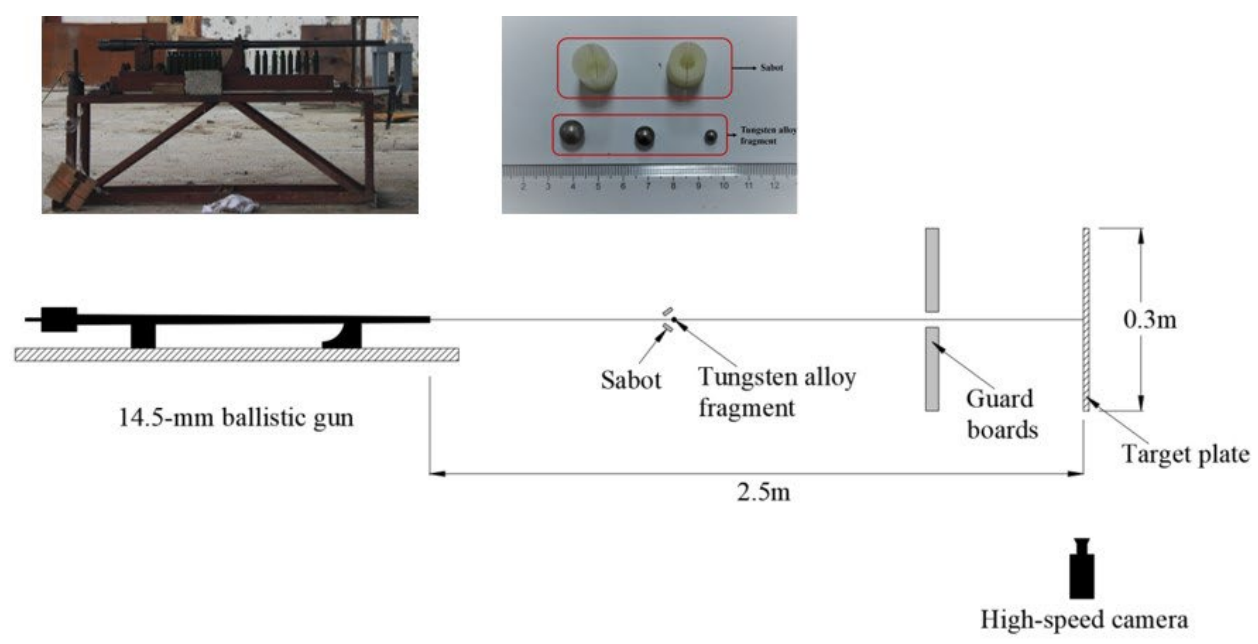

Figure 1 Layout of the ballistic impact testing device

The fragment (tungsten alloy sphere) was placed into the sabot to ensure its sealed state during launch and to control the initial velocity of the fragment. By adjusting the amount of propellant charge, a fragment velocity of $1000 \mathrm{~m} / \mathrm{s}$ could be achieved. Upon exiting from the muzzle, the fragment was separated from the sabot and hit the target plate through the hole of a guard board. The sabot was then intercepted by the guard boards.

The tests were performed on $300 \mathrm{~mm} \times 300 \mathrm{~mm}$ carbon fiber target plates with different thicknesses (Figure 2). The material properties were as follows: fiber volume fraction $\omega=60 \%$, density $\rho_{t}=1.56 \mathrm{~g} / \mathrm{cm}^{3}$, Poisson's ratio $v=0.3$, interlaminar shear strength $\sigma_{t}=86 \mathrm{MPa}$, tensile strength $E=2300 \mathrm{MPa}$, and tensile modulus $G=140 \mathrm{GPa}$. Tungsten alloy spheres with diameters of 5 and $8 \mathrm{~mm}$ were used as prefabricated spherical fragment penetration elements to carry out the armor piercing tests.

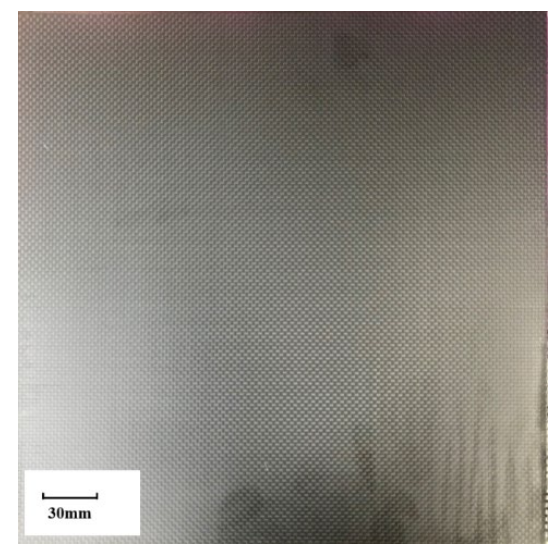

Figure 2 Carbon fiber composite target plate

\subsection{Test facility}

Ballistic resistance performance tests were performed using tungsten alloy spherical fragments with different diameters on carbon fiber composite target plates of different thicknesses. Following the experimental data processing scheme for the tests described above, we obtained the corresponding ultimate penetration velocity. The specific results are shown in Table 1. 
Table 1 Test value of ultimate penetration velocity

\begin{tabular}{cccc}
\hline Order number & Fragment diameters $/ \mathbf{d}(\mathbf{m m})$ & Target thickness $/ \mathbf{h}(\mathbf{m m})$ & \multicolumn{2}{c}{ Ultimate penetration velocity } \\
$\mathbf{l} \mathbf{v}_{\boldsymbol{t}}(\mathbf{m} / \mathbf{s})$
\end{tabular}

The results in Table 1 show that when the fragment diameters were the same, the ultimate penetration velocity of the tungsten alloy spheres hitting the carbon fiber composite target plate increased as the thickness of the target plate increased. When the target plate thicknesses were the same, the ultimate ballistic velocity decreased as the fragment diameter increased. To display the relationship between the ballistic ultimate velocity, fragment diameter, and thickness of the target plate more intuitively, the results were plotted with $\mathrm{h} / \mathrm{d}$ on the $\mathrm{x}$-axis and the ballistic ultimate velocity on the $y$-axis, as shown in Figure 3. There was a linear relationship between $\mathrm{h} / \mathrm{d}$ and the ballistic ultimate velocity.

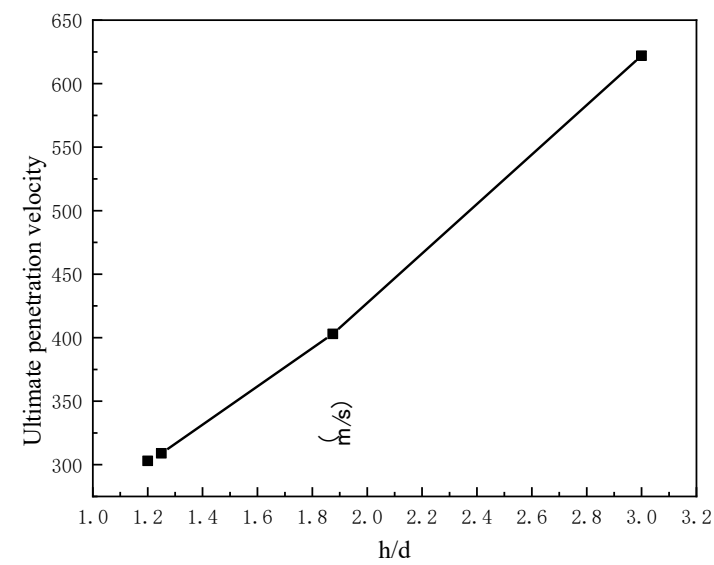

Figure 3 Variation of the experimental $\mathrm{h} / \mathrm{d}$ values in the ballistic ultimate velocity test of tungsten alloy spherical fragments penetrating a carbon fiber target plate target plate

To observe the variation of the penetration energy for fragments with different velocities penetrating the same carbon fiber target plate, penetration tests were conducted at multi-gradient velocities. The test results are shown in Table 2. Under the same projectile and target conditions, the velocity of the fragment striking the target was positively correlated with the energy required to penetrate the target became.

Table 2 Ballistic impact test results of carbon fiber composite target

\begin{tabular}{cccccc}
\hline Order number & $\begin{array}{c}\text { Fragment } \\
\text { diameters(mm) }\end{array}$ & $\begin{array}{c}\text { Target } \\
\text { thickness(mm) }\end{array}$ & $\begin{array}{c}\text { The velocity of } \\
\text { striking the } \\
\text { target(m/s) }\end{array}$ & $\begin{array}{c}\text { The velocity of exiting } \\
\text { the target(m/s) }\end{array}$ & Penetration energy(J) \\
\hline $2-1$ & 5 & 15 & 645 & 110 & 235.28 \\
$2-2$ & 5 & 15 & 682 & 204 & 246.69 \\
$2-3$ & 5 & 15 & 716 & 261 & 258.94 \\
$2-4$ & 5 & 15 & 717 & 314 & 242.02 \\
$2-5$ & 5 & 15 & 731 & 295 & 260.57 \\
$2-6$ & 5 & 15 & 851 & 458 & 299.66 \\
$2-7$ & 5 & 15 & 851 & 438 & 310.09 \\
$2-8$ & 8 & 10 & 310 & 10 & 229.04 \\
$2-9$ & 8 & 10 & 355 & 195 & 239.60 \\
$2-10$ & 8 & 10 & 383 & 302 & 259.26 \\
$2-11$ & 8 & 10 & 465 & 355 & 298.29 \\
$2-12$ & 8 & 10 & 494 & 405 & 281.56 \\
$2-13$ & 8 & 10 & 540 & 517 & 304.38 \\
$2-14$ & 8 & 10 & 648 & 364.12 \\
\hline
\end{tabular}




\section{ANALYSIS OF FRAGMENT PENETRATION PROCESS}

We do not intend to guide the way you want to write your paper, but a section RESULTS is nearly always present in all $t$ The process of a tungsten sphere penetrating a carbon fiber composite target plate involves complex physical and mechanical processes accompanied by the propagation of elastoplastic waves, micro-deformation of the tungsten sphere, local deformation of the target plate, and stress transients (Pei SX et al.,1993). Based on certain assumptions and using dimensional analysis, we established a simplified model to describe the penetration behavior of the carbon fiber target plate.

To facilitate the establishment of the formula, we made the following simplifications of the collision process: (1) all thermal effects were neglected, (2) the spherical fragment was treated as a rigid body and its deformation was ignored, and (3) the fragment trajectories were assumed to be straight lines.

Before the 20th century, the engineering calculation model of fragment penetrating target was established by polynomial fitting method. However, the calculation process of this kind of method is complex, lack of corresponding physical meaning, and can not well describe the influence of a single physical parameter on the calculation results. As a tool to determine the relationship between variables in complex processes or physical phenomena, dimensional analysis can better reflect the laws of physical phenomena than traditional methods.

The process of a spherical fragment penetrating a target plate involves a number of factors. The main factors and their dimensions under the MKS (meter-kilogram-second) system are shown in Table 3. Through analysis of the various factors, we established a dimensionless equation.

Table 3 main factors and dimensions

\begin{tabular}{|c|c|c|}
\hline Variable name & Code name & Primary dimension \\
\hline Fragment diameter & $d$ & $\mathrm{~L}$ \\
\hline Fragment material density & $\rho_{f}$ & $M L^{-3}$ \\
\hline Fragment velocity & v & $L T^{-1}$ \\
\hline Target thickness & $\mathrm{h}$ & $\mathrm{L}$ \\
\hline Target material density & $\rho_{\mathrm{t}}$ & $M L^{-3}$ \\
\hline Shear strength of target plate & $\sigma_{\mathrm{t}}$ & $M L^{-1} T^{-2}$ \\
\hline Penetration energy & E & $M L^{2} T^{-2}$ \\
\hline
\end{tabular}

Based on the parameters given above, the formula of the ultimate penetration velocity of the fragment is as follows:

$v_{t}=f\left(h, d, \rho_{f}, \rho_{t}, \sigma_{t}\right)$

where vt is the ultimate penetration velocity. The equation contains three independent dimensions, and $d$, $\rho f$, and $\sigma t$ may be chosen as the base quantities to measure the quantities in the equation. Hence, the following relationship is satisfied:

$$
\frac{v_{t} \cdot \rho_{f}^{0.5}}{\sigma_{t}^{0.5}}=f\left(\frac{h}{d}, 1,1, \frac{\rho_{t}}{\rho_{f}}, 1\right)
$$

Expanding the function $f$, we have the following:

$$
\frac{v_{t} \cdot \rho_{f}^{0.5}}{\sigma_{t}^{0.5}}=\left(\frac{h}{d}\right)^{a} \cdot\left(\frac{\rho_{t}}{\rho_{f}}\right)^{b}
$$

Reorganizing the two sides of the equation, we arrive at an equation for the ultimate penetration velocity of the fragment: 
$v_{t}=\left(\frac{h}{d}\right)^{a} \cdot\left(\frac{\rho_{t}}{\rho_{f}}\right)^{b} \cdot \frac{\sigma_{t}^{0.5}}{\rho_{f}^{0.5}}$

where $\mathrm{a}$ and $\mathrm{b}$ are undetermined constants.

Taking the logarithm of both sides of Eq. (3) yields the following:

$\ln \left(\frac{v_{t} \cdot \rho_{f}^{0.5}}{\sigma_{t}^{0.5}}\right)=a \ln \left(\frac{h}{d}\right) \cdot b \ln \left(\frac{\rho_{t}}{\rho_{f}}\right)$

Letting

$$
\left\{\begin{array}{l}
X=\ln \left(\frac{h}{d}\right) \\
Y=\ln \left(\frac{\rho_{t}}{\rho_{f}}\right) \\
Z=\ln \left(\frac{v_{t} \cdot \rho_{f}^{0.5}}{\sigma_{t}^{0.5}}\right)
\end{array}\right.
$$

Eq. (4) becomes

$Z=a X+b Y$

Substituting the test data in Table 1 into Eq. (7), we obtain: $a=0.7998$ and $b=-0.5395$, i.e.,

$v_{t}=\left(\frac{h}{d}\right)^{0.7998} \cdot\left(\frac{\rho_{t}}{\rho_{f}}\right)^{-0.5395} \cdot \frac{\sigma_{t}^{0.5}}{\rho_{f}^{0.5}}$.

The energy required for the fragment to penetrate the target plate depends on the velocity of the penetrating fragment. Based on the form of the formula for the ultimate penetration velocity and dimensional analysis, a formula was established for the penetration energy. Table 1 shows the main factors involved in the fragment penetration process of the target plate, together with their dimensions in MKS units.

Based on the given parameters, the equation showing the change of the penetration energy as a function of the change of the velocity is as follows:

$\Delta E=f\left(h, d, \rho_{f}, \rho_{t}, \Delta v, \sigma_{t}\right)$

This formula contains four independent dimensions, $d, \rho t, \sigma t$, and $\Delta v$, which are extracted as the basic quantities to measure each quantity in the formula, where $\Delta \mathrm{v}=\mathrm{v}-\mathrm{vt}, \Delta \mathrm{E}=\mathrm{E}-\mathrm{Et}$, and $\mathrm{Et}$ is the target penetration energy at the ultimate penetration velocity. Thus, the following expression is obtained:

$\frac{\Delta E^{2}}{\rho_{t} d^{6} \sigma_{t} \Delta v^{2}}=f\left(\frac{h}{d}, 1,1, \frac{\rho_{t}}{\rho_{f}}, 1,1\right)$

Expanding the function $f$ yields the following: 
$\frac{\Delta E^{2}}{\rho_{t} d^{6} \sigma_{t} \Delta v^{2}}=\left(\frac{h}{d}\right)^{x} \cdot\left(\frac{\rho_{t}}{\rho_{f}}\right)^{y}$

Reorganizing the two sides of the equation, we obtain

$\Delta E=\sqrt{\left(\frac{h}{d}\right)^{x} \cdot\left(\frac{\rho_{t}}{\rho_{f}}\right)^{y} \cdot \rho_{t} d^{6} \sigma_{t} \cdot \Delta v}$

where $\mathrm{x}$ and $\mathrm{y}$ are constants to be determined.

The curve showing the fragment penetration energy as a function of the fragment velocity will pass through the point ( $\mathrm{vt}, \mathrm{Et}$ ), where Et is the energy at the fragment's ultimate velocity. Thus, the fragment penetration energy formula can be written as follows:

$E-E_{t}=\sqrt{\left(\frac{h}{d}\right)^{x} \cdot\left(\frac{\rho_{t}}{\rho_{f}}\right)^{y} \cdot \rho_{t} d^{6} \sigma_{t}} \cdot\left(v-v_{t}\right)$

Reorganizing both sides of the formula, we have the following:

$\left\{\begin{array}{l}E=A \cdot v+B \\ A=\sqrt{\left(\frac{h}{d}\right)^{x} \cdot\left(\frac{\rho_{t}}{\rho_{f}}\right)^{y} \cdot \rho_{t} d^{6} \sigma_{t}} \\ B=E_{t}-A \cdot v_{t}\end{array}\right.$

Equation (14) shows that under certain conditions of the target, A and B are constants, and the fragment energy needed to penetrate the target increases as the fragment velocity increases. By processing the data in Table 2 using Eq. (14), we obtained the relationship between the penetration energy and the velocity of the fragment, as shown in Figure 4. Table 4 shows the fitting coefficients and the fitting accuracy for different target systems.

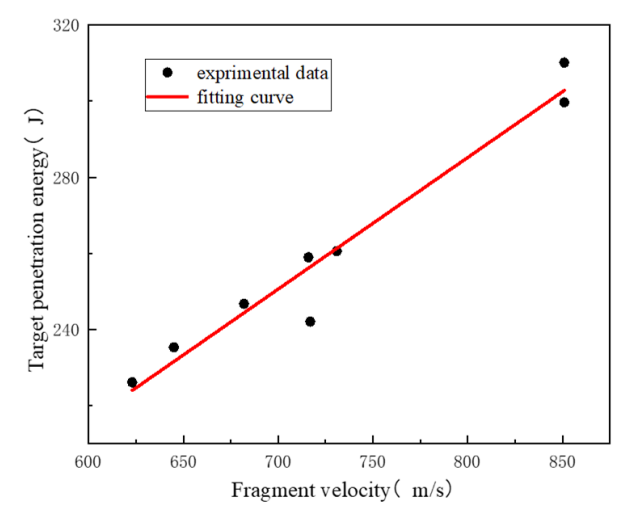

a.

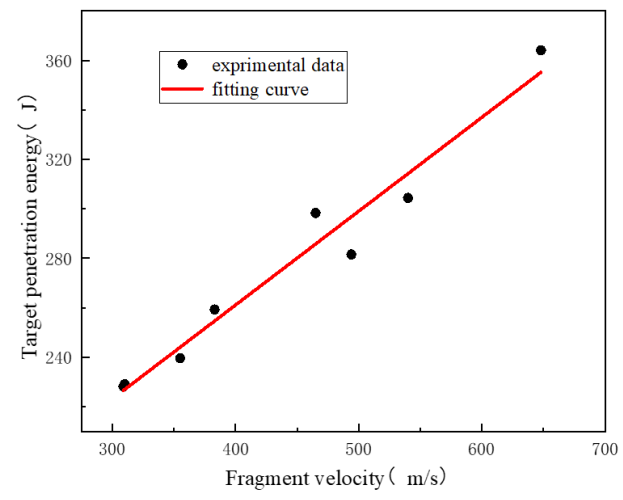

b

Figure 4 Changes of penetration energy with fragment velocity: (a) Condition 1 (5-mm-diameter fragment, 15-mm-thick target plate) and (b) Condition 2 (8-mm-diameter fragment, 10-mm-thick target plate)

Figure 4 shows that, as the velocity of the fragment increased, the energy of the fragment increased accordingly, and the gap between the ultimate penetration energy and the test value of the fragment's penetration energy increased. 
This indicates that when a fragment impinges on the target plate with a velocity greater than the ultimate penetration velocity, the discrepancy between the excess velocity calculated from the ultimate penetration energy and the observed excess velocity will increase as the fragment velocity increases. Under Condition 1, when the fragment velocity was increased by $30 \%$, the discrepancy between the penetration energy and the ultimate penetration energy increased by $32 \%$. In Condition 2, when the fragment velocity increased by $50 \%$, the discrepancy between the penetration energy and the ultimate penetration energy increased by $28 \%$.

The above comparison of the ultimate penetration energy and the test data of the penetration energy showed that the incident velocity of the fragment had a greater impact on the energy required to penetrate the target. The ultimate penetration velocity of the fragment was therefore of limited use in characterizing the bullet-proof performance of the material. In comparison, it is better to use the fragment penetration energy formula to characterize the bullet-proof performance of the material.

Table 4 Fitting coefficient and fitting accuracy of the same missile target system

\begin{tabular}{|c|c|c|c|}
\hline \multirow{2}{*}{ Missile target system } & \multicolumn{2}{|c|}{ Fitting coefficient } & \multirow{2}{*}{ Fitting accuracy } \\
\hline & A & B & \\
\hline 1 & 0.34569 & 8.60265 & 95.21 \\
\hline 2 & 0.37937 & 109.39909 & 95.97 \\
\hline
\end{tabular}

The fitting coefficients in Table 4 show that, for different target systems, the fitting coefficient increased as the ratio of the fragment diameter and target plate thickness increased. This is consistent with the overall change of the projectile and target system shown in Figure 5. In addition, the fitting accuracies for the different target and projectile systems were $95 \%$ or better, indicating that it was reasonable to use Eq. (14) to fit the target penetration energy data and that the formula met the engineering calculation requirements.

After obtaining the A value for the two conditions, it was determined that $x=3.0088$ and $y=-0.3030$. Thus,

$$
\left\{\begin{array}{l}
E=A \cdot v+B \\
A=\sqrt{\left(\frac{h}{d}\right)^{3.0088} \cdot\left(\frac{\rho_{t}}{\rho_{f}}\right)^{-0.3030} \cdot \rho_{t} d^{6} \sigma_{t}} \\
B=E_{t}-A \cdot v_{t}
\end{array}\right.
$$

\section{EXPERIMENTAL VERIFICATION}

To verify the ultimate penetration velocity and penetration energy of tungsten alloy spherical fragments penetrating a carbon fiber composite target plate, perforation tests were conducted on carbon fiber target plates of different thicknesses using fragments with diameters of 5,8 , and $10 \mathrm{~mm}$. The velocities of the fragments were measured using a high-speed camera.

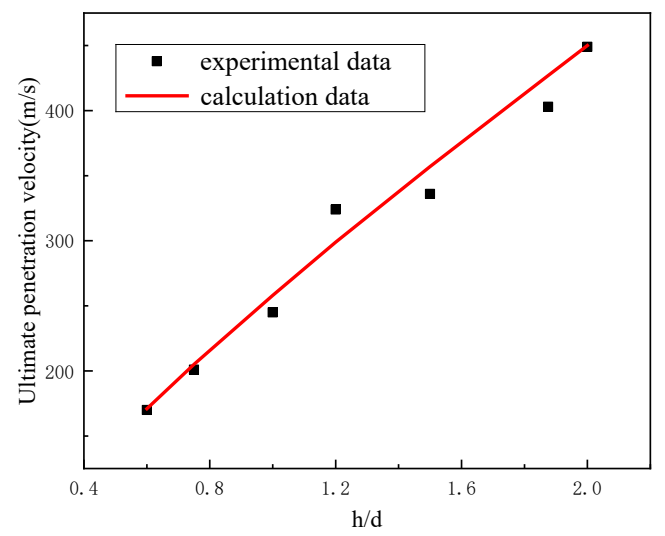

Figure $\mathbf{5}$ Comparison of the experimental and calculated values of the ballistic ultimate velocities of tungsten alloy spherical fragments penetrating carbon fiber composite target plate 
Since Eqs. (8) and (15) were obtained from fitting analysis of the experimental results based on dimensional analysis, they are therefore applicable for the penetration of carbon fiber composite target plates by tungsten alloy spherical fragments. The pertinent parameter ranges are $\mathrm{v}<1000 \mathrm{~m} / \mathrm{s}$ and $0.4<\mathrm{h} / \mathrm{d}<3.3$. The test data are shown in Tables 5 and 6 . The experimental values are compared with the calculated values of equation 8 and equation 16 (Mi SS et al.,2005) in Table 5.

$$
v_{t}=\left[2 k \frac{\sigma_{t}}{m} h^{\frac{3}{2}} S^{\frac{3}{4}} \frac{\rho_{t}}{\rho_{f}^{0.1}}\right]^{\frac{1}{2}}
$$

Where: $k$ is the experimental coincidence coefficient, and the value of spherical fragment $k$ is $0.002 ; m$ is the mass of fragment $(\mathrm{kg}) ; \mathrm{S}$ is the fragment projection area $\left(\mathrm{m}^{2}\right)$.

Table 5 Test value of ultimate penetration velocity

\begin{tabular}{|c|c|c|c|c|c|c|c|}
\hline \multirow{2}{*}{$\begin{array}{l}\text { Order } \\
\text { number }\end{array}$} & \multirow{2}{*}{$\begin{array}{c}\text { Fragment } \\
\text { diameters }(\mathrm{mm})\end{array}$} & \multirow{2}{*}{$\begin{array}{c}\text { Target } \\
\text { thickness }(\mathrm{mm})\end{array}$} & $\begin{array}{c}\text { Ultimate } \\
\text { penetration } \\
\text { velocity }\end{array}$ & \multirow{2}{*}{$\begin{array}{l}\text { Calculated } \\
\text { value of } \\
\text { equation } \\
8(\mathrm{~m} / \mathrm{s})\end{array}$} & \multirow[t]{2}{*}{ Error(\%) } & \multirow{2}{*}{$\begin{array}{c}\text { Calculated } \\
\text { value of } \\
\text { equation } \\
16(\mathrm{~m} / \mathrm{s})\end{array}$} & \multirow[t]{2}{*}{ Error(\% } \\
\hline & & & $\begin{array}{c}\text { Experimental } \\
\text { values }(\mathrm{m} / \mathrm{s})\end{array}$ & & & & \\
\hline $3-1$ & 5 & 10 & 449 & 450 & -0.22 & 184 & 59.02 \\
\hline $3-2$ & 8 & 6 & 201 & 205 & -1.99 & 88 & 56.21 \\
\hline $3-3$ & 10 & 6 & 170 & 171 & -0.58 & 74 & 56.47 \\
\hline $3-4$ & 10 & 10 & 245 & 258 & -5.31 & 109 & 55.51 \\
\hline $3-5$ & 10 & 15 & 336 & 357 & -6.25 & 148 & 55.95 \\
\hline
\end{tabular}

Table 6 Ballistic impact test results of carbon fiber composite target

\begin{tabular}{|c|c|c|c|c|c|c|c|}
\hline \multirow{2}{*}{$\begin{array}{c}\text { Order } \\
\text { number }\end{array}$} & \multirow{2}{*}{$\begin{array}{c}\text { Fragment } \\
\text { diameters }(\mathrm{mm})\end{array}$} & \multirow{2}{*}{$\begin{array}{c}\text { Target } \\
\text { thickness }(\mathrm{mm})\end{array}$} & \multirow{2}{*}{$\begin{array}{l}\text { The velocity of } \\
\text { striking the } \\
\text { target }(\mathrm{m} / \mathrm{s})\end{array}$} & \multirow{2}{*}{$\begin{array}{c}\text { The velocity of } \\
\text { exiting the } \\
\operatorname{target}(\mathrm{m} / \mathrm{s})\end{array}$} & \multicolumn{2}{|c|}{ Penetration energy(J) } & \multirow[b]{2}{*}{ Error(\%) } \\
\hline & & & & & $\begin{array}{l}\text { Experimental } \\
\text { values }\end{array}$ & $\begin{array}{c}\text { Calculated } \\
\text { values }\end{array}$ & \\
\hline $4-1$ & 5 & 10 & 507 & 144 & 137.65 & 128.66 & -6.98 \\
\hline $4-2$ & 5 & 10 & 561 & 246 & 148.07 & 138.80 & -6.67 \\
\hline $4-3$ & 5 & 10 & 598 & 305 & 154.11 & 145.75 & -5.73 \\
\hline $4-4$ & 5 & 10 & 622 & 331 & 161.54 & 150.26 & -7.50 \\
\hline $4-5$ & 10 & 6 & 222 & 137 & 142.20 & 149.86 & 5.11 \\
\hline $4-6$ & 10 & 6 & 223 & 140 & 140.40 & 150.11 & 6.46 \\
\hline $4-7$ & 10 & 6 & 278 & 205 & 164.30 & 163.62 & -0.41 \\
\hline $4-8$ & 10 & 6 & 326 & 260 & 180.23 & 175.41 & -2.74 \\
\hline $4-9$ & 10 & 6 & 347 & 289 & 171.89 & 180.56 & 4.80 \\
\hline
\end{tabular}

The data in Table 5, Table 6 shows that for the tungsten alloy spherical fragment and carbon fiber composite target plate system, the average relative error between calculated value of equation 8 and experimental values of the ballistic ultimate velocity was $2.87 \%$, and the maximum single term error was $6.25 \%$, the average relative error between calculated value of equation 16 and experimental values of the ballistic ultimate velocity was $56.63 \%$, and the maximum single term error was $59.02 \%$. It could be seen from the error analysis that the calculation model established in this paper was more accurate. The mean of the relative error of the calculated and experimental values of the fragment penetration energy was $5.15 \%$. The largest single term error was $7.50 \%$, and the error values were all less than $10 \%$, which meets engineering application requirements. 


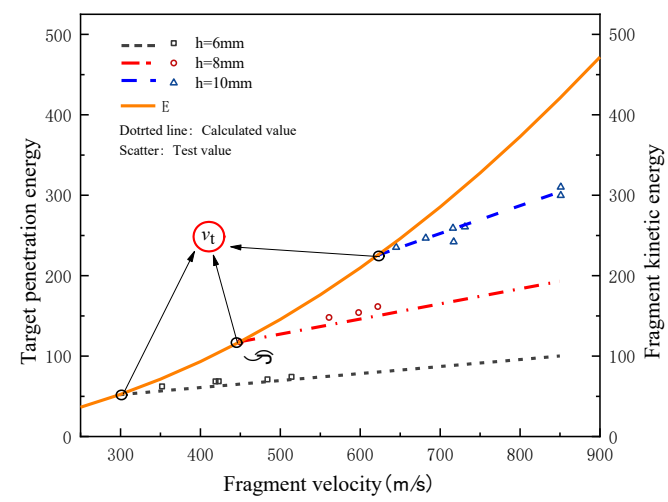

Figure 6 Target penetration energy of 5-mm fragments penetrating target plates of different thicknesses

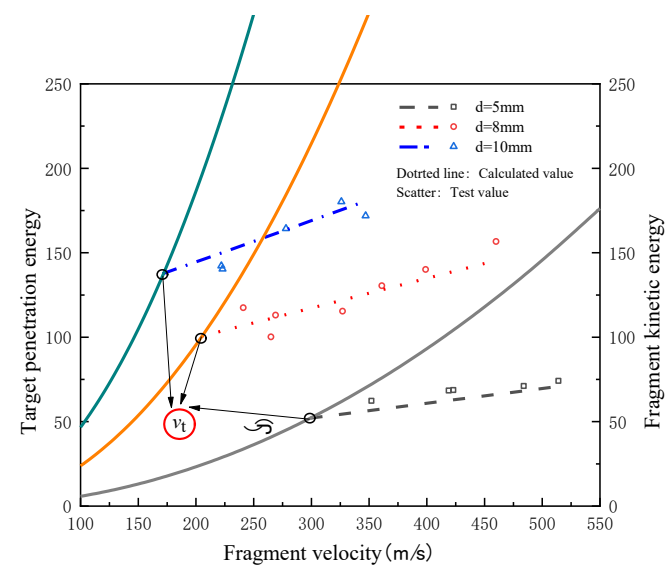

Figure 7 Penetration energy of a 6-mm-thick target plate by fragments of different diameters

The intersection of the curve of the fragment penetration energy versus velocity and the fragment kinetic energy curve is the ultimate penetration point. At this point, the abscissa is the ultimate penetration velocity and the ordinate is the ultimate penetration energy. When the velocity of the fragment was less than the ultimate fragment penetration velocity, the energy for penetrating the target was greater than the kinetic energy of the fragment, so the fragment could not penetrate the target plate. When the fragment velocity was greater than the ultimate penetration velocity, the kinetic energy of the fragment was greater than the energy required to penetrate the target, so the fragment penetrated the target. The region where the two lines intersect corresponds to the region of remaining energy after the fragment penetrated the target. Figure 6 shows that, when the fragment fully penetrated the target plate with the same incident velocity, the greater the thickness of the target plate was, the greater the fragment energy required for penetration became. Figure 7 shows that when the target plate was fully penetrated by fragments of different sizes, the fragment size was positively correlated with the energy required for penetrate target penetration. Furthermore, when the target plate was fully penetrated by fragments of different sizes but with the same kinetic energy, the fragment diameter was positively correlated with the energy required for penetration.

\section{ANALYSIS OF DAMAGE MODE OF CARBON FIBER TARGET PLATE}

The experiments showed that the damage process of a target plate impacted by a fragment involved multi-staged failure modes, comprising mainly three stages (Shi CX,2016): delamination, compressive shear, and tensile damage. Before delamination occurred, the target plate was in the elastic phase. When the fibers were delaminated from the resin matrix, the target plate entered the damage phase, fiber breakage occurred, and the target plate then suffered from shear failure. When the fragment reached the exit surface of the target plate, the fibers on the back surface of the target plate were damaged by stretching, and the back surface bulged outward to form conical deformation. Figure 8 shows the penetration process. 


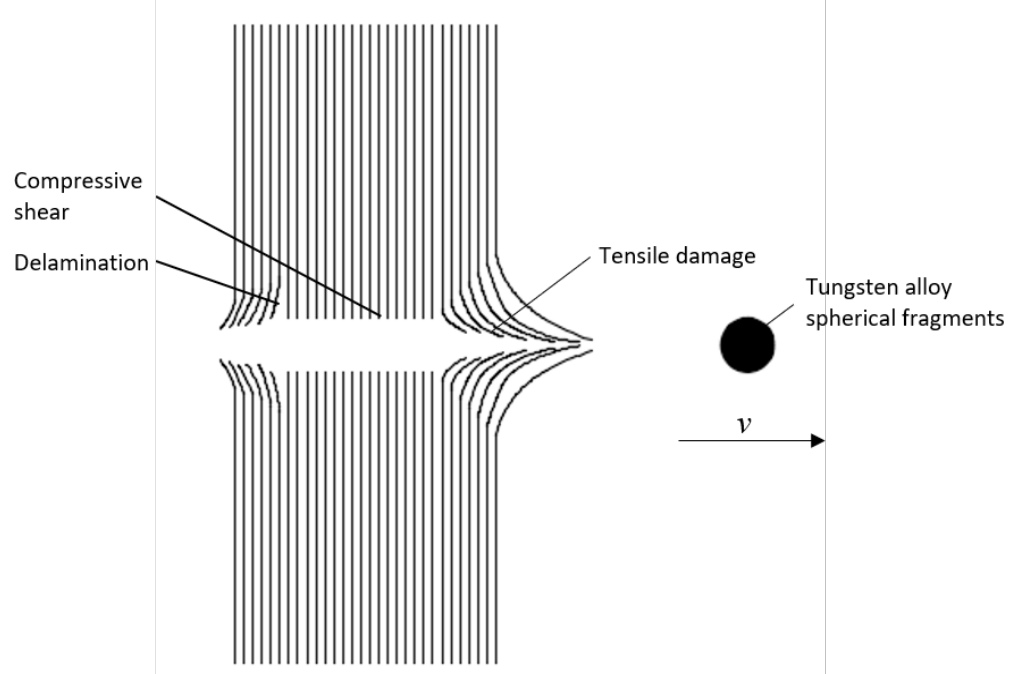

Figure 8 Interaction process of spherical fragments and carbon fiber target plate

We investigated the impact damage mode of the carbon fiber composite target experimentally. Figure 9 shows the damage mode of a $15-\mathrm{mm}$ target plate impacted by a $10-\mathrm{mm}$ fragment at different velocities in the experiment. When the tungsten alloy spherical fragment struck the carbon fiber target plate, the composite material was subjected to compressive stress. The load was transferred to the resin matrix via carbon fibers, causing the resin matrix to crack under the stress load. In the penetration process, a certain amount of heat was generated by the contact of the projectile and the target, and the fibers were softened. Because of the high elongation and high toughness of the fibers, the composite underwent considerable deformation before it failed. The shape of the bulge on the back surface of the target plate changed from irregular to rhombic, and the size of the bulge on the front and back surfaces of the target plate gradually increased. As the velocity of the fragment increased, the bulge on the front surface of the target plate became round again, and the bulge on the back surface of the target plate gradually became smaller. This was because, during the penetration of the high-velocity fragment, the fibers were torn instantly under the action of the force, and only a small portion of the force was transmitted to the resin matrix. As a result, the front surface only formed a round-shaped bulge. As the fragment approached the back surface of the target plate, its velocity had decreased. The bulge on the back surface still had a rhombic shape, and the size of the bulge gradually decreased as the velocity of the fragment increased.
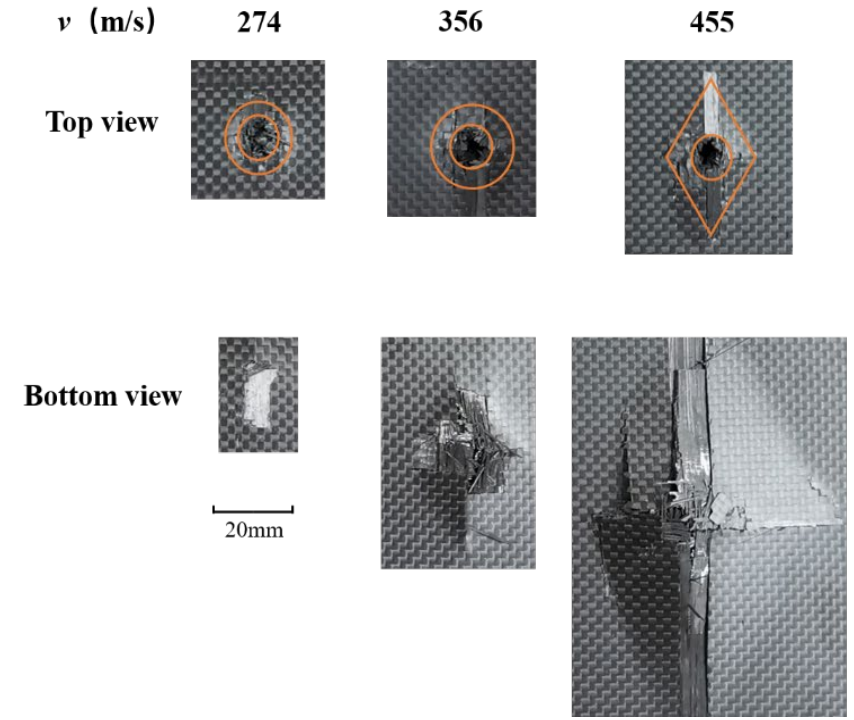

470

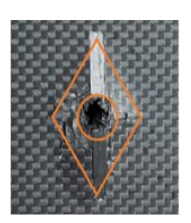

538
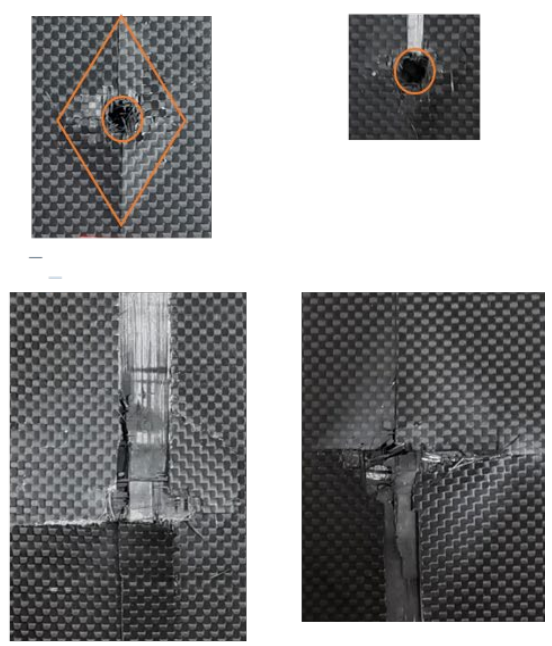

Figure 9 Damage patterns observed in the experiment

As the target plate was penetrated by the fragment, the target plate formed resistance to the projectile perforation through various damage and energy absorption mechanisms. Ignoring the heat generated by the friction between the fragment and the target plate, the energy of the penetrating fragment would be transferred from the fragment to the 
target plate. Therefore, the kinetic energy and the velocity of the penetrating fragment decreased after piercing the target plate. Based on the above analysis of the penetration process, the damage and failure modes of the carbon fiber composite target plate were mainly delamination, fiber shear, and tensile failure. Therefore, the microscopic energy absorption mechanisms mainly included fiber pull-out, debonding, stress redistribution, crack bridging, and matrix plastic deformation (Zhang SY,1992). These are described below.

In the fiber pull-out mechanism, the fiber broke after being impacted. Since the fracture surface did not coincide with the crack surface, the composite material could not break until the fractured fibers were pulled out. The pull-out process needed to first disrupt the interface between the fiber and the matrix, which involved the absorption of a certain amount of energy.

In the debonding mechanism, since the fracture strain of the fiber was greater than that of the matrix, the fiber continued to be pulled longer after the matrix material had cracked. In the meantime, the fracture of the matrix caused shedding of the load and a decrease in the matrix deformation. This led to interfacial debonding due to a greatly increased shear stress at the fiber matrix interface.

In the stress redistribution mechanism, before the fiber broke, since the elastic modulus of the fiber was much greater than that of the matrix, the force on the matrix was extremely small compared to the force on the fiber. After the fiber suddenly broke, the load on the fiber was transferred to the matrix, causing stress redistribution.

In the crack bridging mechanism, during the process of forming a crack bridge, a matrix crack first opened. This required work to be performed in the formation of relative displacement by overcoming the interfacial shear stress between the fiber and the matrix. This energy transfer process was very similar to that of that of the fiber pull-out mechanism. In addition, when the fiber broke, it triggered another stress redistribution process. Therefore, the formation process of a crack bridge was accompanied by the process of fiber pullout and stress redistribution.

In the matrix plastic deformation mechanism, when brittle fibers were adhesively bonded to a ductile matrix, the fibers at the front edge of the crack often broke. The matrix deformed plastically due to the increased force. This was followed by local necking, and finally, total separation.

\section{CONCLUSION}

In this work, impact tests were conducted using tungsten spheres of various diameters and carbon fiber composite target plates of different thicknesses. Quantitative data were obtained for the ballistic impact properties of carbon fiber composites. Through dimensional analysis, a penetration energy equation was obtained for the fragment penetration of carbon fiber target plates. The following conclusions were obtained.

In the same fragment and target system, with spherical fragments fully penetrating the target plate, the energy required for the fragment to penetrate the target plate increased with increasing incident fragment velocity. In the lowvelocity stage, there was a linear relationship between the penetration energy and the incident fragment velocity. For target plates fully penetrated by fragments of different sizes with the same incident velocity, the fragment diameter was positively correlated with the required energy for the fragment to penetrate the target. Through dimensional analysis, an equation was obtained for the penetration energy required for the tungsten alloy spherical fragments to penetrate the carbon fiber composite target plates. The calculated and experimental values showed good agreement. This equation meets engineering requirements, with an error within $10 \%$. Analysis of the damage modes of the target plate showed that the main damage modes of the carbon fiber composite target plate penetrated by tungsten alloy spherical fragments were delamination, compressive shear, and tensile damage. The microscopic energy absorption mechanisms mainly included fiber pull-out, disbanding, stress redistribution, crack bridging, and plastic deformation of the matrix.

Author's Contribuitions: Methodology, WB Li, D Hong; Writing - original draft, D Hong, N Jiang and Y Zheng; Writing review \& editing, D Hong and Y Zheng; Experiment, D Hong, N Jiang and Y Zheng.

Editor: Marcílio Alves.

\section{References}

Cantwell WJ, Morton J. Comparison of the low and high velocity impact response of cfrp [J]. Composites, 1989, 20(6): 545551. 
Chai G B, Zhu S. A review of low-velocity impact on sandwich structures[J]. Proceedings of the Institution of Mechanical Engineers, Part L: Journal of Materials: Design and Applications, 2011, 225(4): 207-230.

Devarajan B, Kapania RK. Thermal buckling of curvilinearly stiffened laminated composite plates with cutouts using isogeometric analysis [J]. Composite Structures, 2020, 238.

Hazell P J, Kister G, Stennett C, et al. Normal and oblique penetration of woven CFRP laminates by a high velocity steel sphere[J]. Composites Part A: Applied Science and Manufacturing, 2008, 39(5): 866-874.

Kinloch A J, Maxwell D L, Young R J. The fracture of hybrid-particulate composites[J]. Journal of Materials Science Letters, 1985, 20(11): 4169-4184.

Kinloch A J, Taylor A C. Mechanical and fracture properties of epoxy/inorganic micro- and nano-composites[J]. Journal of Materials Science Letters, 2003.

Kinloch A J, Taylor A C. The mechanical properties and fracture behaviour of epoxy-inorganic micro- and nano-composites[J]. Journal of Materials Science Letters, 2006, 41(11): 3271-3297.

Koubaa S, Mars J, Wali M, et al. Numerical study of anisotropic behavior of Aluminum alloy subjected to dynamic perforation[J]. International journal of impact engineering, 2017, 101(MAR.): 105-114.

Mars J, Chebbi E, Wali M, et al. Numerical and experimental investigations of low velocity impact on glass fiber-reinforced polyamide[J]. Composites Part B: Engineering, 2018a.

Mars J, Said L B, Wali M, et al. Elasto-Plastic Modeling of Low-Velocity Impact on Functionally Graded Circular Plates[J]. International Journal of Applied Mechanics, 2018b, 10(4).

Meffert B, Milewski G. Aramid liners as armor augmentation[C]. Reliability \& Maintainability Symposium, 2002.

Mi SS, He JB, Zhang XE, et al. Equivalent Target and Terminal Velocity of Fragments in Battle Damage Simulation [J]. Acta Armamentarii, 2005, 26 (5): 605-608

Miglani J, Devarajan B, Kapania R K. Thermal buckling analysis of periodically supported composite beams using Isogeometric analysis[C]. 2018 AIAA/ASCE/AHS/ASC Structures, Structural Dynamics, and Materials Conference, 2018.

Morye S S, Hine P J, Duckett R A, et al. Modelling of the energy absorption by polymer composites upon ballistic impact[J]. Composites ence \& Technology, 2000, 60(14): 2631-2642.

Pandya K S, Pothnis J R, Ravikumar G, et al. Ballistic impact behavior of hybrid composites[J]. Materials \& Design, 2013, 44: 128-135.

Pei SX, Wu XM, Sun T, Zhang GW. Research on the penetration of multilayered target by tungsten spheres. Journal of Projectiles, Rockets, and Guidance, 1993, (2): 23-30.

Ping S A, Wbl A, Yu Z A, et al. The constitutive behavior of Ti-5Al-3V-2Cr-2Fe under high-velocity impact: Experimental, modeling, and validation[J], 2019, 811: 151946-.

Razali N, Sultan M, Mustapha F, et al. Impact damage on composite structures - a review[J]. Int J Eng Sci (IJES), 2014, 3(7): 820.

Shaktivesh, Nair N S, Sesha Kumar C V, et al. Ballistic impact performance of composite targets[J]. Materials \& Design, 2013, 51: 833-846

Shi CX. Study of Penetration Mechanism of Fragment Resistant Fiber-Reinforced Composite Laminates [dissertation]. 2016.

Wambua $\mathrm{P}$, Vangrimde $\mathrm{B}$, Lomov $\mathrm{S}$, et al. The response of natural fibre composites to ballistic impact by fragment simulating projectiles[J]. Composite Structures, 2007, 77(2): 232-240.

Wang S, Zhi XQ, Wang X. Experimental study of ultimate penetration velocity of small tungsten fragment penetrating bulletproof equipment. Proceedings of OSEC First Ordnance Engineering Conference, 2017.

Wen H M. Predicting the penetration and perforation of FRP laminates struck normally by projectiles with different nose shapes[J]. Composite Structures, 2000, 49(3): 321-329.

Withers J C, Storm R S, Shapovalov V, et al. COMPOSITE ARMOR TILE BASED ON A CONTINUOUSLY GRADED CERAMIC-METAL COMPOSITION AND MANUFACTURE THEREOF[J], 2011. 
Zhang F, Xu W, Yin YX, et al. Application of Chinese-made BHM3 carbon fiber in satellite structures. Aerospace Manufacturing Technology, 2015, 193(5): 30-33.

Zhang SY. Mechanical Properties of Composite Structure. 1992.

Zhang ZY, Dai F. Application of composite materials in armored tank vehicles. Plastics, 2000, (3): 38-42.

Zhu G, Goldsmith W, Dharan C K H. Penetration of laminated Kevlar by projectiles-II. Analytical model [J]. Int J Solids Struct, 1992, 29: 421-36.

Zukas J. Impact dynamics[J]. New York: John Wiley \& Sons, 1990. 